\title{
THE EFFECTS OF FLOW-INHOMOGENEITIES ON MOLECULAR CLOUD FORMATION: LOCAL VERSUS GLOBAL COLLAPSE
}

\author{
Jonathan J. Carroll-Nellenback ${ }^{1}$, Adam Frank ${ }^{1}$, and Fabian Heitsch ${ }^{2}$ \\ ${ }^{1}$ Department of Physics and Astronomy, University of Rochester, Rochester, NY 14620, USA; johannjc@ pas.rochester.edu \\ ${ }^{2}$ Department of Physics and Astronomy, University of North Carolina Chapel Hill, Chapel Hill, NC 27599, USA \\ Received 2013 April 4; accepted 2014 March 28; published 2014 July 1
}

\begin{abstract}
Observational evidence from local star-forming regions mandates that star formation occurs shortly after, or even during, molecular cloud formation. Models of molecular cloud formation in large-scale converging flows have identified the physical mechanisms driving the necessary rapid fragmentation. They also point to global gravitational collapse driving supersonic turbulence in molecular clouds. Previous cloud formation models have focused on turbulence generation, gravitational collapse, magnetic fields, and feedback. Here, we explore the effect of structure in the flow on the resulting clouds and the ensuing gravitational collapse. We compare two extreme cases, one with a collision between two smooth streams, and one with streams containing small clumps. We find that structured converging flows lead to a delay of local gravitational collapse ("core formation"). Hence, the cloud has more time to accumulate mass, eventually leading to a strong global collapse, and thus to a high core formation rate. Uniform converging flows fragment hydrodynamically early on, leading to the rapid onset of local gravitational collapse and an overall low core formation rate. This is also mirrored in the core mass distribution: the uniform initial conditions lead to more low-mass cores than the clumpy initial conditions. Kinetic $\left(E_{k}\right)$ and gravitational energy $\left(E_{g}\right)$ budgets suggest that collapse is only prevented for $E_{k} \gg E_{g}$, which occurs for large scales in the smooth flow, and for small scales for the clumpy flow. Whenever $E_{k} \approx E_{g}$, we observe gravitational collapse on those scales. Signatures of chemical abundance variations evolve differently for the gas phase and for the stellar population. For smooth flows, the forming cloud is well mixed, while its stellar population retains more information about the initial metallicities. For clumpy flows, the gas phase is less well mixed, while the stellar population has lost most of the information about its origin.
\end{abstract}

Key words: instabilities - ISM: clouds - methods: numerical - stars: formation - turbulence

\section{INTRODUCTION}

The concept of flow-driven cloud formation (VázquezSemadeni et al. 1995; Ballesteros-Paredes et al. 1999; Hartmann et al. 2001) can explain two observational constraints on how molecular clouds form stars, derived from local star-forming regions: first, all local molecular clouds are observed to be forming stars, and second, the stellar age spreads are on the order of 1-2 Myr, several times shorter than cloud crossing times (see summary in Hartmann et al. 2001; Ballesteros-Paredes \& Hartmann 2007). The first constraint suggests that star formation sets in immediately (or even during) molecular cloud formation. The second constraint is trivially fulfilled in a scenario where the clouds themselves form in large-scale "converging" flows. The immediate ("rapid") onset of star formation in the forming clouds and the fact that the star formation efficiency is only a few percent (Evans et al. 2009) mandates that the clouds are highly structured: local collapse must set in before global collapse can overwhelm the dynamics (Heitsch et al. 2008b).

The notion of cloud formation in converging flows has led to a series of numerical experiments investigating the physical processes relevant for the rapid fragmentation and for the control of the star formation efficiency. Despite different initial and boundary conditions, the models agree on the following results. (1) Rapid fragmentation is induced by strong radiative losses during the flow collision (possibly even by thermal instability if the clouds form from atomic gas), and by dynamical instabilities (Hueckstaedt 2003; Audit \& Hennebelle 2005; Vázquez-Semadeni et al. 2006; Heitsch et al. 2008a). (2) Turbulence in molecular clouds is a natural result of the dynamical instabilities during the cloud formation (Koyama \& Inutsuka 2002; Heitsch et al. 2005, 2006; Vázquez-Semadeni et al. 2006, 2007; Inoue \& Inutsuka 2009, 2012). At later stages of the cloud evolution, global gravitational collapse leads to large-scale motions (Vázquez-Semadeni et al. 2007; Heitsch et al. 2008b). Yet, the fragmented nature of the evolving cloud makes a clear distinction between infall and turbulence difficult (Heitsch et al. 2009) - they may be two consequences of the same force (gravity). (3) Strong, non-linear density contrasts can also be driven by self-gravity in finite clouds, due to geometry (or "edge") effects (Burkert \& Hartmann 2004; Pon et al. 2011). (4) Although the rapid fragmentation can keep the star formation efficiency low, eventually, feedback or cloud dispersal is needed to prevent a large percentage of the gas to participate in gravitational collapse (Vázquez-Semadeni et al. 2010).

The most obvious difference in the simulation results is the morphology of the forming clouds. All models use some mechanism to break the symmetry in the colliding flowsotherwise, the flow collision would result in a plane-parallel shock. Models with small-scale perturbations ("noise") in the velocities (e.g., Vázquez-Semadeni et al. 2007; Banerjee et al. 2009) tend to lead to extremely flattened clouds with a strong ring due to the gravitational edge effect (Burkert \& Hartmann 2004; Hartmann \& Burkert 2007) in finite, sheet-like clouds. If the velocity perturbations are imposed on larger scales (e.g., as turbulent power spectrum; Hennebelle et al. 2008; Inoue \& Inutsuka 2012) or if the collision interface between the two flows is perturbed (Heitsch et al. 2008b), turbulent structures form that are not necessarily coherent when viewed from different directions (Heitsch et al. 2009).

To understand better the effect of initial conditions on the clouds forming in the flow collisions, we present a simple experiment, comparing two (otherwise identical) cloud formation 
simulations, one with a smooth inflow, and one with a clumpy inflow of identical mass. The experiment is also motivated by the analysis of Pringle et al. (2001), suggesting that cloud formation out of warm atomic gas would require time and length scales too large to be feasible (see also McKee \& Ostriker 2007). This problem is solved by the realization that the flow collision is three-dimensional, allowing gravitational collapse and accretion along the dimensions perpendicular to the flows, and thus circumventing the one-dimensional limit on column density accumulation (Heitsch et al. 2008b). Yet, Pringle et al. (2001) suggest that clumpy flows could reduce the accumulation and molecule formation time scale (especially if the clumps are already molecular). Clumpy structure in pre-shock gas has also been shown to drive turbulent motions in the post-shock gas (Dobbs \& Bonnell 2007). Here, we will test, what effects a clumpy flow has on the resulting cloud and star formation process.

\section{METHOD, INITIAL CONDITIONS, AND PARAMETERS}

We use the adaptive-mesh-refinement (AMR) code AstroBEAR 2.0 to solve the equations of hydrodynamics including self-gravity and equilibrium cooling. For a detailed discussion of AstroBEAR, see Carroll-Nellenback et al. (2013). Poisson's equation is solved with HYPRE (Falgout \& Yang 2002). We used a non-split Corner Transport Upwind (CTU) integrator following Gardiner \& Stone (2008), and the sink particle implementation discussed by Federrath et al. (2010).

We performed two simulations of $40 \mathrm{pc}$ diameter flows with a mean density $n=1.0 \mathrm{~cm}^{-3}$, colliding head-on at $v_{0}=8.25 \mathrm{~km} \mathrm{~s}^{-1}$ for a period of $30 \mathrm{Myr}$. The flows collide in the $y-z$ plane within a box that is $62.5 \times 100 \times 100 \mathrm{pc}^{3}$ in size. The flow setup is similar to that used by Heitsch et al. (2008b), i.e., the flows are entering through the lower and upper $x$-boundary plane, leading to a continuous mass growth within the simulation domain.

We use a base grid of $40 \times 64 \times 64$ cells with five additional levels of refinement for an effective resolution of $1280 \times$ $2048 \times 2048$ and a physical cell size of $\approx 0.05$ pc. Since typical protostellar core radii range around $0.1 \mathrm{pc}$, we interpret the sink particles as gravitationally bound cores, and will refer to the sinks as cores henceforth. The flows combine to give a mass flux of $\dot{M}=665 M_{\odot} \mathrm{Myr}^{-1}$ and a ram pressure of $P_{\text {ram }}=10,472 \mathrm{~K} \mathrm{~cm}^{-3}$. We used an ideal equation of state at $\gamma=5 / 3$, with a mean particle mass $\chi=1.27 \mathrm{amu}$ and a parameterized cooling function $\mathcal{S}$ that includes heating terms consistent with Inoue $\&$ Inutsuka (2008) though modified to give lower temperatures $10 \mathrm{~K}$ at higher densities $\left(n>10^{3} \mathrm{~cm}^{-3}\right)$ to account for UV shielding (R. J. Tanner \& F. Heitsch, in preparation):

$$
\begin{aligned}
\mathcal{S}= & n(-\Gamma+n \Lambda) \mathrm{erg} \mathrm{cm}^{-3} \mathrm{~s}^{-1} \\
\Gamma= & 2 \times 10^{-26} \\
\frac{\Lambda}{\Gamma}= & 1.0 \times 10^{7} \exp \left(\frac{-118400}{T+1000}\right) \\
& +1.4 \times 10^{-2} \sqrt{T} \exp \left(\frac{-22.75}{\max [1.0, T-4]}\right) .
\end{aligned}
$$

The combined heating and cooling results in a thermal equilibrium pressure for each density. This curve can be seen in the dashed line of Figure 1. Note the dashed line only extends to densities of $10^{2} \mathrm{~cm}^{-3}$ to avoid confusion at higher densities-but the equilibrium curve can be seen at higher densities in the distribution itself because the thermal timescales are much smaller than any other time scale at those densities, and thus the gas lines up with the equilibrium curve. In the "Smooth" simulation, the inflowing gas has a uniform density of $1.0 \mathrm{~cm}^{-3}$ and a thermal equilibrium pressure of $4931 \mathrm{~K} \mathrm{~cm}^{-3}$. For the "Clumpy" simulation, the mean inflow density is also $1.0 \mathrm{~cm}^{-3}$, yet the flow contains many small clumps of radius $0.55 \mathrm{pc}$ and a density of $n_{c}=15.2 \mathrm{~cm}^{-3}$, placed randomly in a smooth lower background density of $n_{0}=0.25 \mathrm{~cm}^{-3}$. Both the clumps and the low density background are at the same pressure $1714 \mathrm{~K} \mathrm{~cm}^{-3}$, and both are in thermally stable regimes. However, while the low-density background is in thermal equilibrium with respect to heating and cooling processes, the dense clumps are not, and they do cool and compress somewhat before reaching the oppositely directed material. This can be seen in the upper right panel of Figure 1. The high density contrast $\chi=60.8$ between clumps and background results in a filling fraction of $f=0.05$. The clump radius was chosen to be much less than the Jeans length at the clump density and pressure $\left(L_{J}=43.3 \mathrm{pc}\right)$ so that the clumps would be stable against gravitational collapse. In both runs, the interface between oppositely directed material is initially rippled with a random sequence of sines and cosines of amplitude $2 \mathrm{pc}$, wavelengths from $40 \mathrm{pc}$ down to $2.5 \mathrm{pc}$ and power proportional to $k^{-3}$.

\section{RESULTS}

All of the following analysis focuses on a cylindrical region that is $40 \mathrm{pc}$ in diameter and $10 \mathrm{pc}$ thick, centered on the interface between the two flows. This region is outlined in Figures 2 and 3. Figure 1 shows the joint probability distribution in logarithmic density-pressure space for both runs at times 10.1,20.1, and 27.2 Myr.

\subsection{Thermal Properties}

In the Smooth model (left column), material enters the collision region on the equilibrium curve at $1.0 \mathrm{~cm}^{-3}$ which coincides with the peak in the equilibrium cooling curve. As the material collides with the oppositely directed material it is initially compressed adiabatically up to the flow ram pressure at $1.04 \times 10^{4} \mathrm{~cm}^{-3}$. It then cools and compresses onto the thermal equilibrium curve. With time, more material piles up at higher densities. Eventually, self-gravity takes over at the highest densities and compresses this material further, above the ram pressure provided by the flow. At this point, gas collapses and forms a core, or is being accreted by an existing core. The core formation or accretion explains the lack of material at densities above $\approx 10^{4.6} \mathrm{~cm}^{-3}$.

The Clumpy flow, on the other hand (right column), has material entering the collision region at both $0.25 \mathrm{~cm}^{-3}$ and $15.2 \mathrm{~cm}^{-3}$. Additionally, some mixing occurs between the clumps and the background flow which causes the thick band of material below the thermal equilibrium curve. At these densities, the thermal time scales are longer than the dynamical time (see Figure 3 of Heitsch et al. 2008a)—so this material does not equilibrate before colliding with the oppositely directed flow. The low-density background appears to also compress adiabatically though not to as high pressures as the Smooth run. At the interface between the two flows there are three possible types of interactions due to the two densities present in the flow. (1) For background-background collisions, the ram pressure will be one-fourth of that for the Smooth flow. (2) Collisions between background material from one side and 


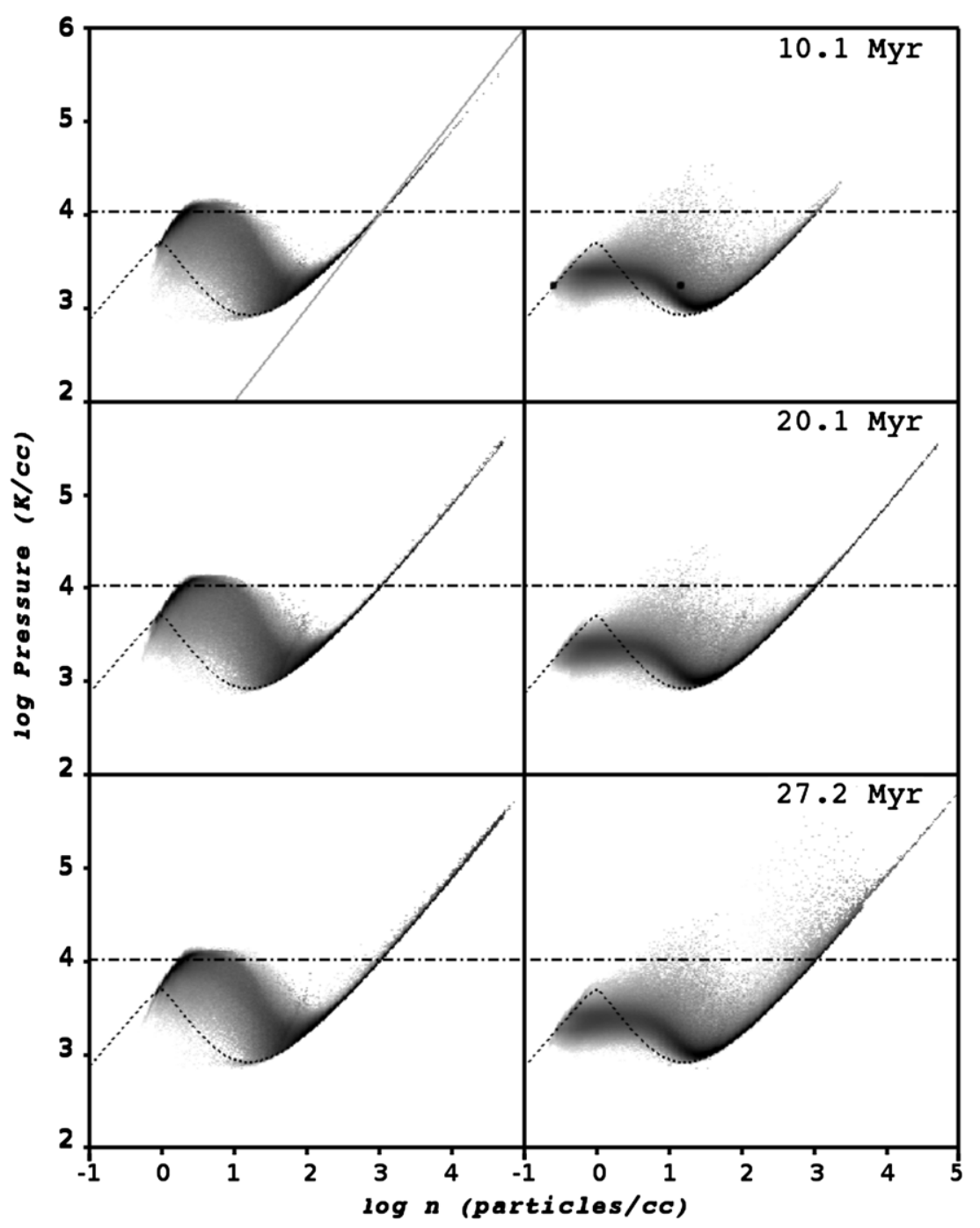

Figure 1. Density-weighted joint probability distribution function for density vs. pressure for the Smooth run (left) and Clumpy run (right), at 10.1, 20.1, and 27.2 Myr. The dot-dashed line indicates the ram pressure of the flows, and the diagonal line in the upper left plot shows $T \equiv 10 \mathrm{~K}$.

clumps from the other will produce bow shocks equivalent to background material running over a stationary clump with velocity $2 v_{0}=16.5 \mathrm{~km} \mathrm{~s}^{-1}$, resulting in a ram pressure equal to that in the Smooth model, at $1.04 \times 10^{4} \mathrm{~K} \mathrm{~cm}^{-3}$. (3) Finally, head-on clump-clump collisions, though rare, can produce pressures 15.2 times the ram pressure in the Smooth model. Yet this material will cool fairly quickly due to the high densities.

\subsection{Morphologies}

Figures 2 and 3 show column densities taken along the flow axis (left) and normal to the flow axis (right) for the Smooth and Clumpy runs, respectively. Also shown are the boundaries of the cylindrical region used for the following analysis. The Smooth run exhibits the usual filamentary structure due primarily to the non-linear thin shell instability (NTSI; Vishniac 1994; Hueckstaedt 2003; Heitsch et al. 2005; Vázquez-Semadeni et al. 2006), and at later times gravity. Also visible is material which has been "splashed" radially outward from the collision region due to the high ram pressures. The NTSI focuses material into various nodes and by $10.1 \mathrm{Myr}$ the first core (solid black square) has formed in one of these nodes (see also Heitsch et al. 2008b). By 20.1 Myr, nine cores have formed throughout the complex and by $27.2 \mathrm{Myr}, 27$ cores have begun to arrange themselves into clusters.

The morphology of the Clumpy run (Figure 3) is quite different from the Smooth run. The view along the flow axis shows that the clumps are confined to be within the stream by a few parsecs and they have a tendency to cluster around the perimeter. This is just an artifact of the clump placing algorithm and can be safely ignored. There also tends to be much less radial splashing than in the Smooth run because the uniform background component prone to being splashed out is only onefourth as dense. The interaction region is much more extended along the flow axis than in the Smooth run. Early on, dense clumps run into the lighter background and travel a distance before being destroyed. The timescale for the clump destruction will be of order the clump crushing time $t_{\mathrm{cc}}=\chi r_{c} / v_{w}$ (Klein et al. 1994) where $v_{w}$ is the "wind velocity" as seen by the clump and $\chi$ is the density contrast. Since the clump is itself traveling into an oppositely directed flow, $v_{w}=2 v_{0}$ and the distance the clump will travel will be of the order of

$$
\mathcal{D}=t_{\mathrm{cc}} v_{0}=\frac{\chi r_{c}}{2}=4.3 \mathrm{pc} \text {. }
$$

If the clump survives for a few clump crushing times, it will travel distances of $\approx 10 \mathrm{pc}$ before being destroyed. This explains 


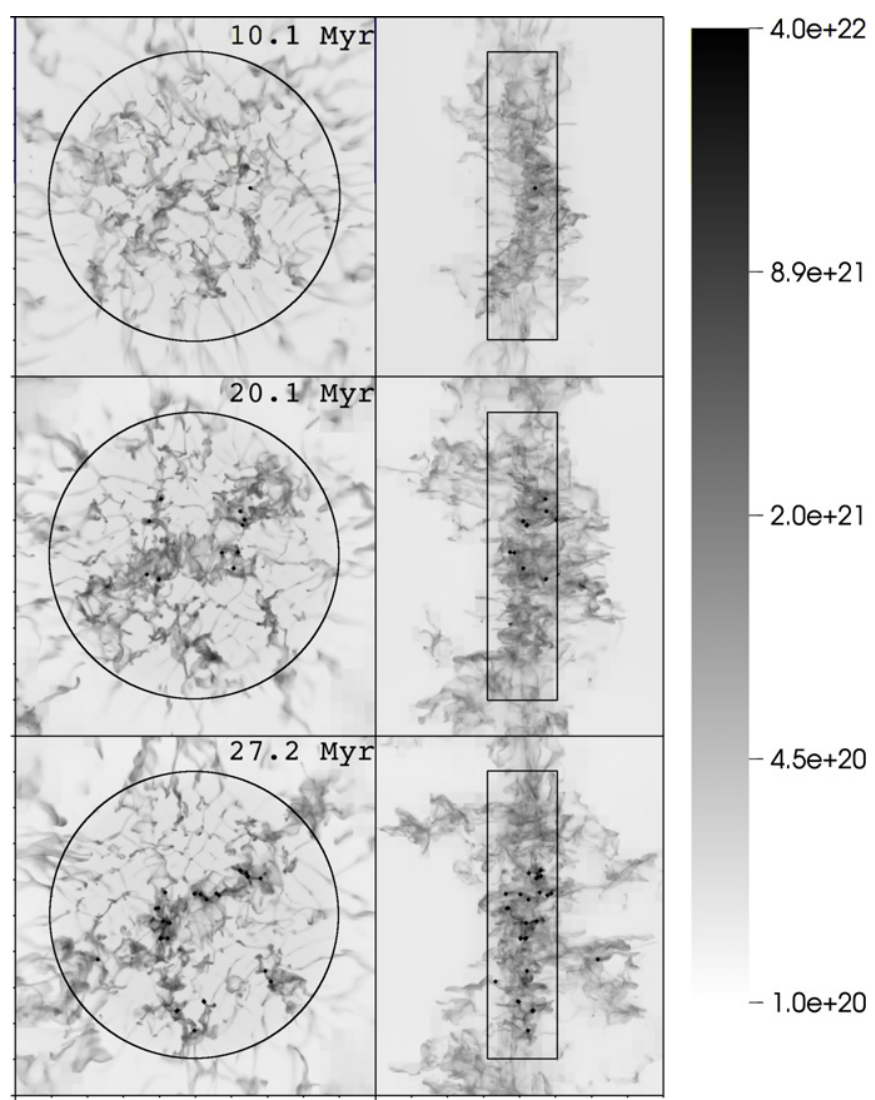

Figure 2. Column density in units of $\mathrm{cm}^{-2}$ projected parallel (left) and perpendicular (right) to flow axis, at 10.1, 20.1, and 27.2 Myr for the Smooth run. Sink particles appear as small black squares. Tick marks are spaced every $5 \mathrm{pc}$ and each panel is $50 \mathrm{pc}$ tall.

the more extended interaction region in the upper right panel of Figure 3. Later in time, the clumps pass through a denser wall of material that has built up and they are also pulled back by gravity - so the extent of the collision region shrinks over time. While the Smooth run has formed nine isolated cores by 20.1 Myr distributed throughout the collision region, the Clumpy run has only just formed a group of three cores near the center of the global potential well. By $27 \mathrm{Myr}$, the entire region is undergoing rapid global collapse and a dense group of 20 cores has formed again near the center of the potential well. The Smooth run has formed 27 cores by the same time.

\subsection{Spectra}

In order to construct power spectra, typically one uses a fast Fourier transform (FFT). However, the FFT requires a uniform grid of points. The process of mapping AMR data onto a fixed grid often leads to spurious signals at wavelengths corresponding to coarser cell sizes depending on the method used to interpolate the data. In AMR simulations, coarse data is often interpolated onto a finer mesh through a process called prolongation. Typically a single coarse cell will be divided into $R^{D}$ finer cells, where $R$ refers to the refinement ratio-or the ratio between cell width-and $D$ is the dimension of the grid. For constant prolongation, these finer cells just inherit the values for density, momentum, etc.. from the coarser parent cell. However, this results in smooth gradients in coarse cells turn into stair steps on finer cells. This stair stepping artificially increases power at the wavelength of the coarser cell. One can do linear interpolation, or higher order interpolation to reduce the effect,
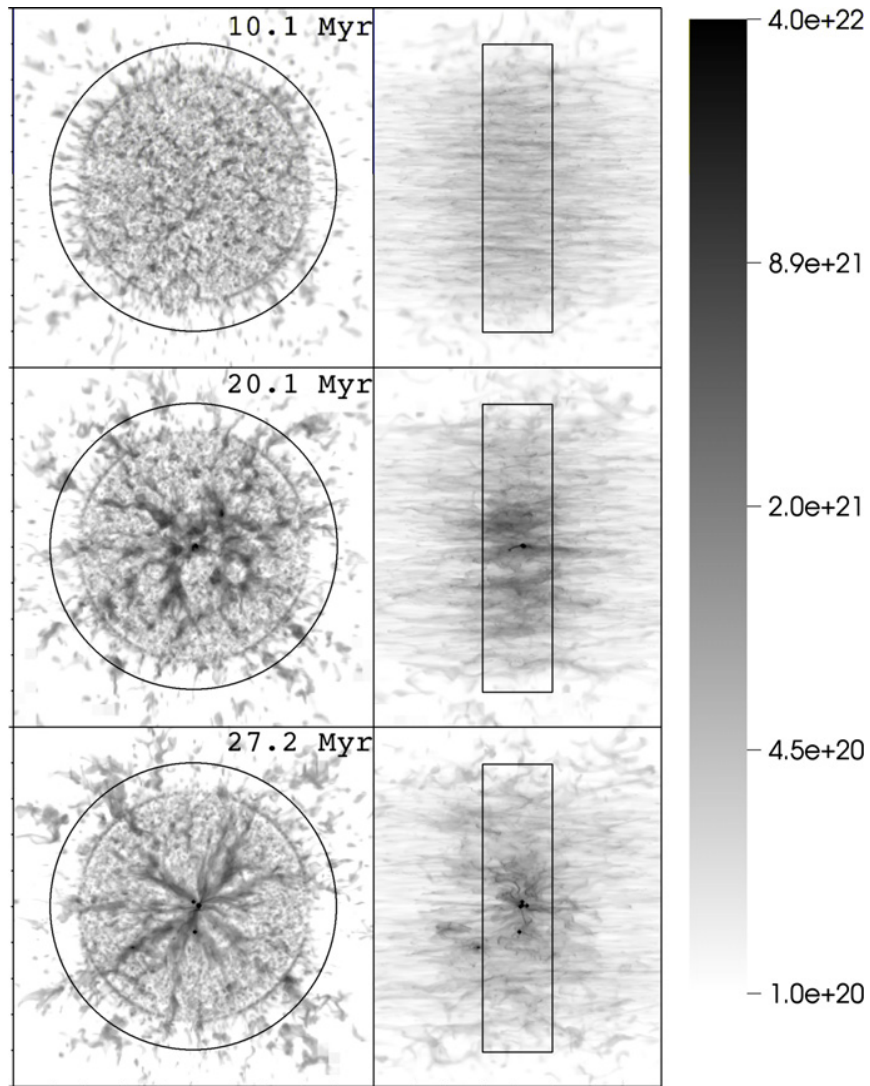

Figure 3. Column density in units of $\mathrm{cm}^{-2}$, projected parallel (left) and perpendicular (right) to flow axis, at 10.1, 20.1, and 27.2 Myr for the Clumpy run. Sink particles appear as small black squares.

but here we prefer to do interpolation in Fourier space-so that the process of interpolating does not introduce any new power in the resulting spectra. The procedure involves first coarsening (averaging) the data everywhere to a uniform coarse grid and then prolongating it to a finer uniform grid using Fourier prolongation described in the Appendix. The finer uniform grid is then updated with existing finer data (where available) and then the process is repeated until we have a uniform grid of points on the finest level of the AMR hierarchy. For details on the prolongation see the Appendix.

The FFT also expects the data to be periodic, however, the physical boundaries are not periodic, and treating them as such introduces a step function at the $x$-boundaries where the inflowing material changes sign. To obtain the power spectrum for a non-periodic velocity field, we introduced a window of $d=40.625 \mathrm{pc}$ in diameter centered on the collision region. The velocity field at a distance $r$ from the center of the collision region is first multiplied by this windowing function before calculating the power spectra:

$$
w(r)= \begin{cases}\cos (\pi r / d) & : r<=d / 2 \\ 0 & : r>d / 2 .\end{cases}
$$

Figure 4 shows spectra of the kinetic and gravitational energy densities, $E_{k}$ and $E_{g}$. The contribution of cores (i.e., sink particles) to the energy densities are not included. Snapshots have been taken at three times, early in the cloud evolution (corresponding to a diffuse CO cloud) at $t=10.1 \mathrm{Myr}$ after simulation start, an intermediate stage $(t=20.1 \mathrm{Myr})$, and a late stage $(t=27.2 \mathrm{Myr})$. In the following discussion, we will distinguish between "large" and "small" scales by considering 

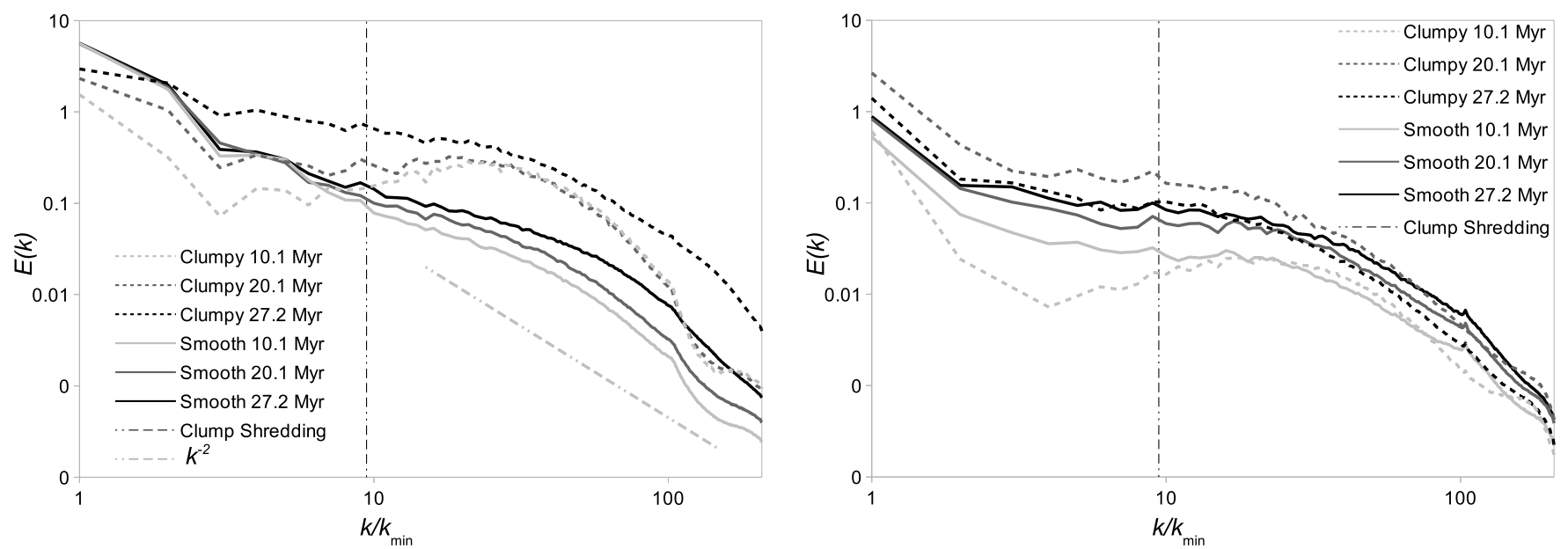

Figure 4. Kinetic energy (left) and gravitational energy spectra (right) for both runs and three times as indicated in the diagram. The vertical line indicates the clump shredding distance (Equation (2)).

scales larger or smaller than the clump crushing distance $\mathcal{D}$ (Equation (2)).

At early times ( $t=10.1 \mathrm{Myr}$, light gray lines), the Clumpy run shows a kinetic and gravitational energy excess on small scales, with $E_{k} \gg E_{g}$. While both runs have the same influx of kinetic energy driven by the flows through the boundaries, the Clumpy run contains most of the kinetic energy in dense clumps on small scales. When these flows collide, the clumps are able to maintain coherence until they have travelled a clump shredding distance (Equation (2)) at which point their kinetic energy is able to dissipate. This clump shredding distance can be thought of as a "driving scale" for the small scale "turbulence" visible in the excess, and it roughly corresponds to the break in the power spectrum, justifying our choice of this scale to separate between "large" and "small" scales.

By 20.1 Myr, the Clumpy run (dark gray, dashed lines) has gained large-scale kinetic energy due to the onset of global collapse mirrored in an increase of the gravitational energy on large scales, with $E_{g} \approx E_{k}$. By 27.2 Myr the Clumpy run has gained kinetic and gravitational energy on all scales due to both the continued global collapse as well as the onset of local collapse. Note that the overall apparent drop in gravitational energy in the Clumpy run between 20.1 and 27.2 Myr is due to the very rapid accretion onto sink particles, following the global collapse (as stated before, the spectra only refer to the energy in the gas phase, not in the sink particles).

The Smooth run (solid lines) shows only a modest increase in the small-scale kinetic and gravitational energy, with $E_{g} \approx E_{k}$ except at very early times on large scales, when $E_{k} \gg E_{g}$. With advancing time, $E_{g}>E_{k}$ for small scales, whereas $E_{g}<E_{k}$ on large scales.

\subsection{Energy Budgets}

The time evolution of the energy spectra highlights a fundamental ambiguity in the interpretation of the kinetic energy. On the one hand, for situations with $E_{k} \approx E_{g}$, the models show gravitational collapse on the respective scales, suggesting that the kinetic energy actually follows the gravitational one, i.e., that the increase in kinetic energy is due to gravitational collapse (see Vázquez-Semadeni et al. 2007; Heitsch et al. 2008b). On the other hand, for situations $E_{k} \gg E_{g}$ (as for small scales in the Clumpy run), the kinetic energy seems to take on an active role preventing gravitational collapse.
We attempt to throw some more light on this ambiguity by following the time evolution of the mean energy densities $\bar{E}_{g}$ and $\bar{E}_{k}$ for large and small scales (Figure 5), where "large" and "small" is defined in Section 3.3. The mean energy densities are derived by averaging over scales $<\mathcal{D}$ (small scales) and $>\mathcal{D}$ (large scales) in the energy spectra.

For the Smooth run (right panel of Figure 5) we note that $\bar{E}_{k} \gg \bar{E}_{g}$ on large scales. This is consistent with the lack of global collapse observed for the Smooth run, and also with the absence of large-scale modes in the energy spectra (Figure 4). However, the small scale gravitational energy becomes comparable to the small scale kinetic energy after 10 Myr which is when we begin to see the formation of isolated cores, indicating local collapse. The Clumpy run on the other hand shows the opposite-the energy is dominated by small scale kinetic energy $\left(\bar{E}_{k} \gg \bar{E}_{g}\right)$ at times for which no local collapse occurs. Only after $20 \mathrm{Myr}$, when on large scales $\bar{E}_{k} \approx \bar{E}_{g}$, do we begin to see global collapse. By $23 \mathrm{Myr}$, much of the gas has been accreted into several large cores near the center of the potential well, which explains the drop in energies.

While both runs begin with the same total kinetic energy, and while they have the same influx of kinetic energy, the Smooth run is much more efficient at dissipating this energy in large coherent shocks resulting in a smaller overall kinetic energy within the collision zone. In the Clumpy run, the density contrast between the clumps and the opposing ambient material leads to a less efficient dissipation of kinetic energy. This excess kinetic energy on small scales suppresses local collapse (remember that the clumps themselves are gravitationally stable) but cannot prevent global collapse-while in the Smooth run, the larger amount of kinetic energy on large scales resists global collapse but not local collapse. Another way to see this is that the shocks in the Smooth run will fragment quickly due to the thermal instability. Yet, the velocity dispersion between the fragments will be small, at least smaller than for the Clumpy run (see Figure 6, right), thus forming structures that are more or less coherent in velocity space. Thus, local collapse is seeded. For the Clumpy run, thermal instability does not play much of a role, and while the clumps can accrete their own mass within a few Myr, they are still (thermally) Jeans-stable. Thus, local collapse is suppressed, while global collapse sets in once enough mass in clumps has been assembled. Note that the excess of small-scale kinetic energy in the clumpy run results from the relative motions of the clumps, not from turbulence within the 

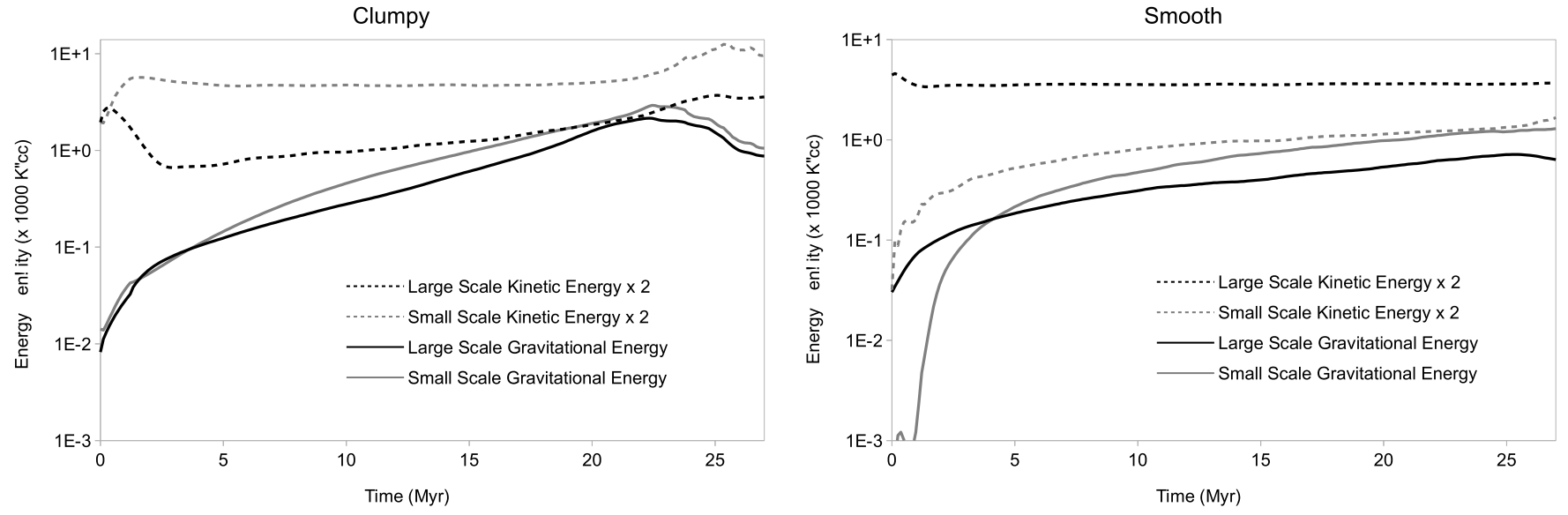

Figure 5. Mean kinetic and gravitational energy densities $\bar{E}_{k, g}$ against time, split between large (>D ; see Equation (2)) and small scales for both the Clumpy run (left) and the Smooth run (right). Small-scale kinetic energy excess in the Clumpy run indicates prevention of local collapse, while $\bar{E}_{g} \approx \bar{E}_{k}$ suggests gravitational collapse (see the text).
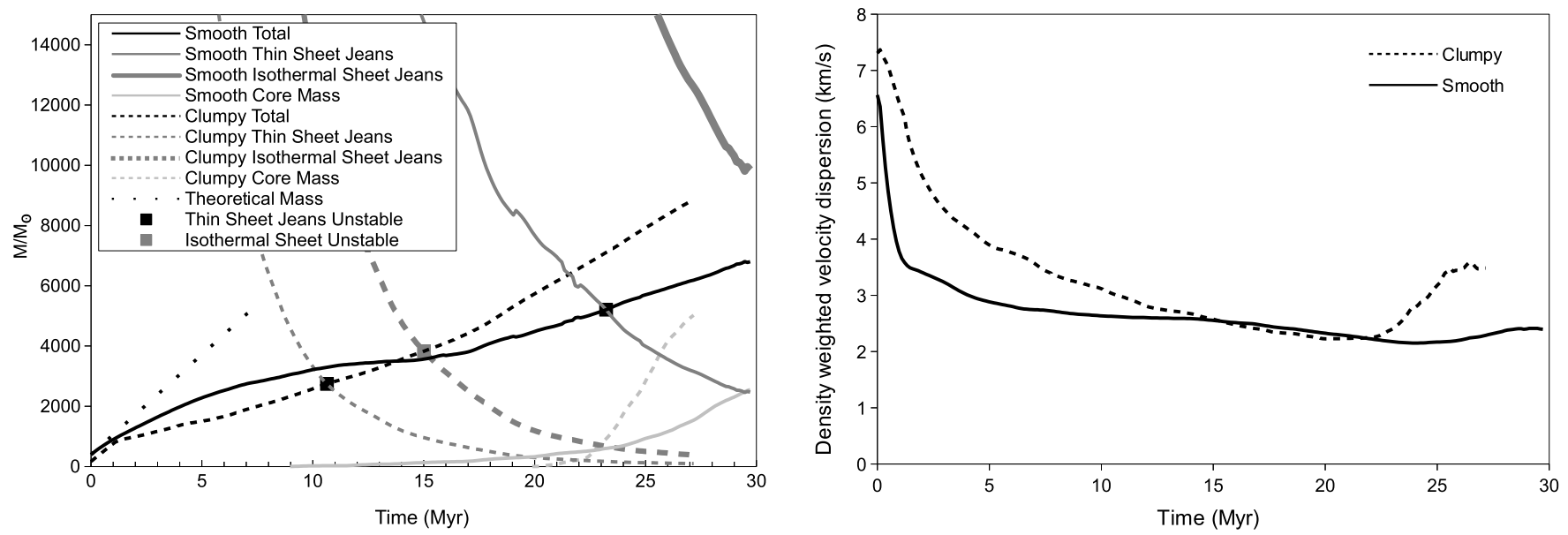

Figure 6. Left: mass history against time for the Clumpy and Smooth run. Black lines indicate total mass (including sinks) in the analysis region, thin/thick dark gray lines trace the average thin/isothermal sheet Jeans mass (Equations (3) and (4)), also in the analysis region, and the light gray lines follow the mass in sinks alone, tracing local collapse. The dotted line stands for the mass accumulation expected from simple sweep-up. Local collapse is suppressed in the Clumpy run until $\approx 20 \mathrm{Myr}$, while the Smooth run forms sinks after $\approx 10 \mathrm{Myr}$. Right: density-weighted velocity dispersion against time, for the Clumpy and Smooth run, again within the analysis region. The Clumpy dispersion is systematically higher until $\approx 15 \mathrm{Myr}$, and it increases again once global collapse sets in at $22 \mathrm{Myr}$.

clumps. The power spectrum shows this as an excess energy content at small scales.

Summarizing, we point out that in our interpretation the fact that $\bar{E}_{k} \approx \bar{E}_{g}$ is not a sign of virialization, and thus balance between gravitational contraction and turbulent support, but actually a natural consequence of gravitational collapse (Ballesteros-Paredes et al. 2011). Only for $\bar{E}_{k} \gg \bar{E}_{g}$ there is enough kinetic energy in the system to prevent gravitational collapse of the highly structured, irregular, and non-linear cloud.

\subsection{Core Formation}

The story of local versus global collapse also can be gleaned from the mass histories (left panel of Figure 6). The mass histories are restricted to the analysis region (see beginning of Section 3). The dotted line in Figure 6 shows the theoretical upper bound using the mass influx $\dot{M}$ through the boundaries. Both runs collect mass at the inflow rate $\dot{M}$ for $t<2$ Myr, after which the growth rate drops. For the Smooth run, material is being splashed radially outwards, and at later times, some of the NTSI fingers develop past the analysis region (see Figure 2). Eventually, after $15 \mathrm{Myr}$, material is falling back in from the edges of the analysis region, increasing the mass collection rate again, i.e., steepening the slope. In the Clumpy run, some of the clumps exit the analysis region on the far side after 2 Myr. The overall mass collection rate slowly increases after that, with material falling back in, and eventually collapsing globally.

The Smooth run begins forming cores at $10 \mathrm{Myr}$, and by $25 \mathrm{Myr}$, the rate of total mass growth and core mass growth have become equal. This implies that material is being accreted by the cores at the same rate it is entering the collision region. The Clumpy run (Figure 3) does not begin to form cores until $20 \mathrm{Myr}$, but then quickly accretes gas at a rate higher than the mass flux into the region. This suggests a degree of global collapse not present in the Smooth run.

To better understand the degree of gravitational instability in both models, we calculate the Jeans mass, averaged over the analysis region. Given the geometry, we compare the Jeans mass for a thin, infinite sheet at a given sound speed $c_{s}$ and column density $\Sigma$,

$$
M_{J 0}=1.17 \frac{c_{s}^{4}}{G^{2} \Sigma}
$$

(Larson 1985), and the critical mass for an isothermal, infinite sheet,

$$
M_{c}=4.67 \frac{c_{s}^{4}}{G^{2} \Sigma}
$$



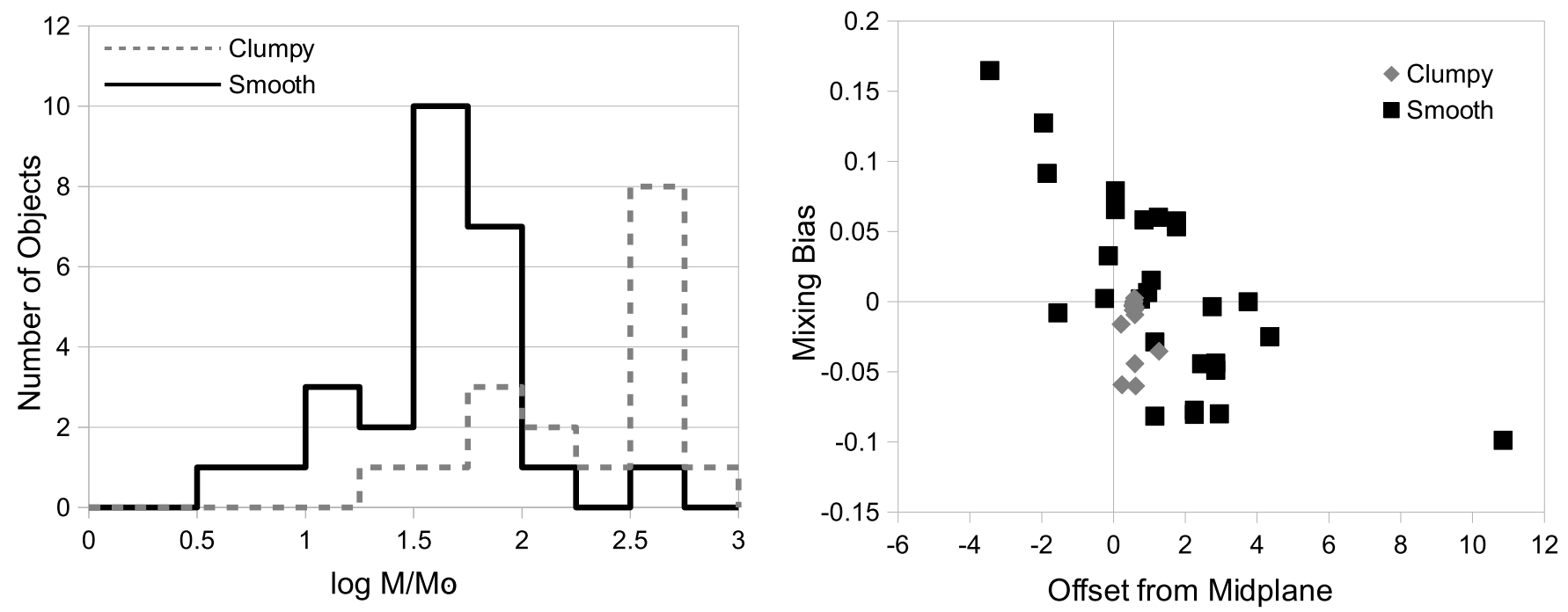

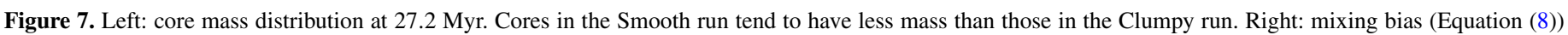
vs. distance of cores to mid-plane, at 27.2 Myr. Smooth cores show a bias, while Clumpy cores are clustered around the origin.

(Larson 1985). Both expressions are obviously an approximation, since our model clouds is neither a perfect sheet, nor infinite. The mass column density $\Sigma$ is averaged over the analysis region, as is the sound speed. The latter is calculated as

$$
c_{s}^{2}=\frac{5}{3} \frac{k_{B}}{\chi} \frac{\int \rho T d V+10 \mathrm{~K} \sum m_{n}}{\int \rho d V+\sum m_{n}},
$$

where the integrals extend over the analysis region and $m_{n}$ refers to the mass of the $n$th core. We also assume that the cores (i.e., the sinks) are at a temperature of $10 \mathrm{~K}$. These critical masses are plotted in the left panel of Figure 6 as well as the locations at which the actual mass becomes theoretically gravitational unstable (large squares).

Since the gas is compressed and thus cools, the Jeans mass will drop with time, as shown in Figure 6 (left panel). It levels out once the minimum temperature of $\approx 10 \mathrm{~K}$ is reached (this is only obvious in the Clumpy run, dashed medium or thick lines, for $t>25 \mathrm{Myr}$ ). The Jeans mass for the Clumpy run is smaller by at least an order of magnitude, because of the clumps at higher densities and lower temperatures. Yet, since these clumps do not form a coherent region with $M>M_{J}$, local gravitational collapse is suppressed until $\approx 20 \mathrm{Myr}$, and sinks form only once global collapse sets in, indicated by the increasing slope of the total mass, black dashed line. The onset of global collapse in the Clumpy run is also visible in Figure 5 (left), and in the velocity dispersions shown in the right panel of Figure 6.

The Smooth run has a substantially larger Jeans mass that does not level out at a minimum within the model run time. Yet, because of the rapid local fragmentation into structures larger than a local Jeans mass, local collapse (and sink formation) sets in at $\approx 10 \mathrm{Myr}$. If the steepening of the total mass curve with time at $\sim 15 \mathrm{Myr}$ is interpreted as a sign of fall-back, and thus global collapse, then the Smooth run shows a substantially weaker signature of global collapse compared to the Clumpy run, consistent with the evolution of the energy budgets and the velocity dispersions.

Note that Equations (3) and (4) refer to the global Jeans mass. This may explain the somewhat counter-intuitive notion of the Smooth run having a larger Jeans mass than the Clumpy run, while it develops gravitational collapse at an earlier time. Since the flow compression in the Clumpy run is less important than in the Smooth run, the Jeans mass of the Clumpy run is largely determined by the cold clumps. For the Smooth run, the flow compression drives the density up and the temperature down, leading to a locally much smaller Jeans mass, hence rapid local fragmentation and collapse. Yet, comparing Figures 2 and 3 at 10.1 Myr shows that in fact the filamentary structures of the Smooth run are less volume-filling than the clumps of the Clumpy run. Thus, the global Jeans mass is a better predictor for the behavior of the Clumpy run than that of the Smooth run.

Figure 7 shows the distribution of core masses for both runs at 27.2 Myr. Most of the cores in the Smooth run have masses $<100 M_{\odot}$, while there is a substantial fraction of cores in the Clumpy run with masses $>100 M_{\odot}$. This is consistent with the findings of Vázquez-Semadeni et al. (2009), who compared "low-mass regions" (LMR) and "high-mass regions" (HMR) in their simulations of flow-driven cloud formation. They found that cores in LMRs tend to have lower masses, while those in the HMR formed due to global collapse of the whole cloud tend to have higher masses. Unlike Vázquez-Semadeni et al. (2009), we do not observe LMRs and HMRs in one and the same simulation. In fact, in our models, the total mass in cores for the Clumpy and Smooth run differ only by 50\%, while the LMR and HMR of Vázquez-Semadeni et al. (2009) seem to have a mass ratio of nearly 10. Nevertheless, the cores in the Clumpy run form at the center of the potential well (Figure 3 ) late in the simulation, i.e., at a time by which global collapse has set in, while the cores in the Smooth run start to form much earlier (Figure 6, light gray lines), at a shallower gravitational potential. That the Smooth core mass distribution is slightly wider than the Clumpy one, would also be consistent with this scenario: with a deepening gravitational potential, more massive cores are formed. Yet, for more definitive statements, we would need better statistics.

It also should be pointed out that we measure the mass in cores at equal times (27.2 Myr), while Vázquez-Semadeni et al. (2009) derive the statistics for their LMRs and HMRs within one and the same simulation at different times. Due to the global collapse and the more massive "seed"-cores, one might expect the mass difference between our Clumpy and Smooth cores to increase further with time.

\subsection{Mixing}

In both runs, material injected from the left and right side was marked with a tracer $\left(\rho_{L}\right.$ and $\left.\rho_{R}\right)$ proportional to the density so 

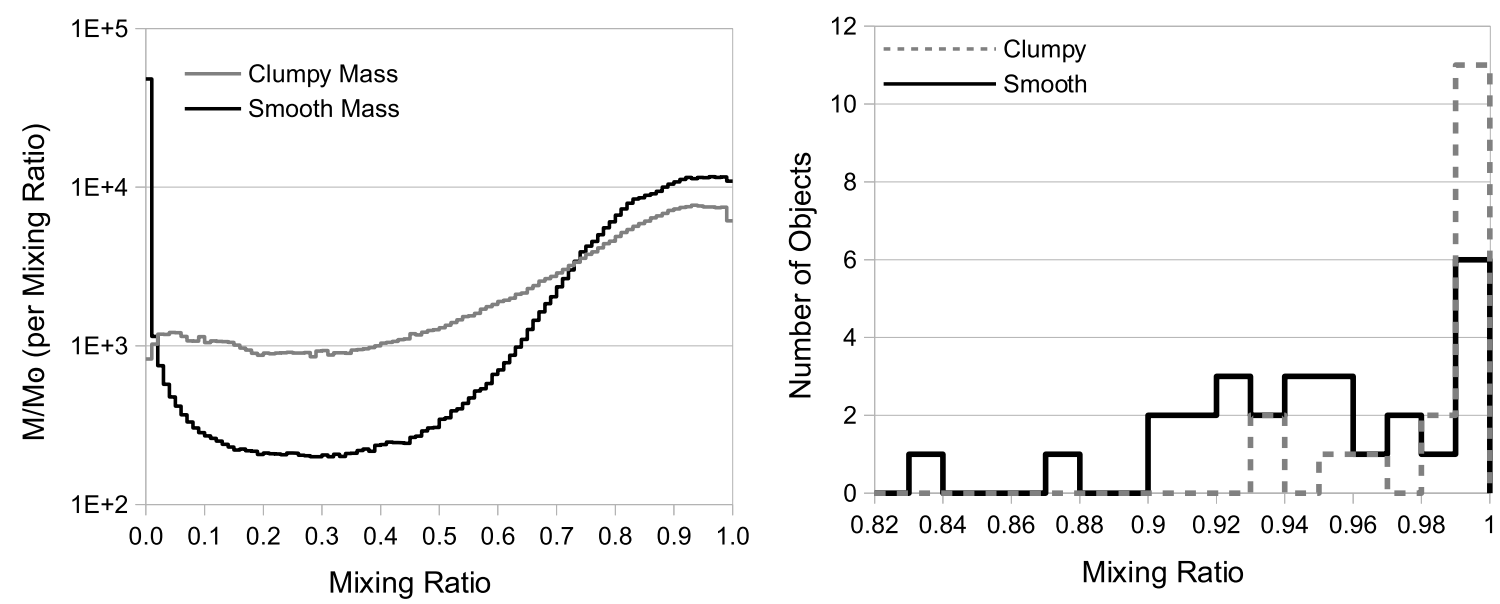

Figure 8. Left: mass-weighted mixing ratio (Equation (7)) for the gas in the analysis region, at 10.1 Myr. Right: mixing ratio of cores at $27.2 \mathrm{Myr}$.

that the amount of mixing could be investigated. We then define a local mixing ratio

$$
\operatorname{MR}=\frac{2 \min \left(\rho_{L}, \rho_{R}\right)}{\max \left(\rho, \rho_{L}+\rho_{R}\right)} .
$$

Thus, $\mathrm{MR}=0$ indicates the presence of only one tracer at a location (or none - as is the case in the ambient medium outside of the flow), and MR = 1 indicates equal amounts of both tracers with no ambient material mixed in. Since we are confining our analysis to the colliding flow region, there should be no ambient material present so $\rho=\rho_{L}+\rho_{R}$ - and the definition is equivalent to

$$
\mathrm{MR}=\frac{2 \min \left(\rho_{L}, \rho_{R}\right)}{\rho_{L}+\rho_{R}} .
$$

The Smooth run shows a higher mass-fraction of well-mixed material (Figure 8, left panel). The mass in the gas phase (i.e., not including the cores/sink particles) is comparable for both runs (see left panel of Figure 6), at around $4 \times 10^{3} M_{\odot}$. The Clumpy run tends to have a more spread out distribution of mixing ratios than the Smooth run. As clumps drive through the opposing stream-they will shed some of their material and provide varying amounts of mixing. In the Smooth case, the flows interact along a thin interface and it is difficult to get unequal amounts of material from either side in the same region.

While the gas in the Smooth run shows a higher mixing ratio (i.e., more uniform composition) than in the Clumpy run, the cores in the Smooth run (Figure 8, right panel) have lower mixing ratios. This can be understood in two ways.

1. In the Smooth run, the NTSI creates nodes that act to funnel material streaming into the "trough" from only one side, while diverting material from the other side. If so, then cores that formed to the left of the collisional mid-plane should be biased towards the right tracer and vice versa. To test this, we define a mixing bias as

$$
\mathrm{MB}=\frac{\rho_{R}-\rho_{L}}{\rho_{R}+\rho_{L}}
$$

and plot it against the core's offset from the $y-z$ mid-plane shown in the right panel of (Figure 7). Indeed, cores with negative offsets (left of mid-plane) tend to have a higher value for the mixing bias so they have more right tracer and are comprised of material primarily from the right side, and vice versa. Note that this bias is absent in the cores formed in the Clumpy run, whose cores are clustered around 0.
2. In the Clumpy run, instantaneous mixing in the gas is suppressed in the absence of a strong NTSI: the clumps just plow through the oncoming gas. Thus, mixing in the gas phase will be generally low. Yet, as Banerjee et al. (2009) discussed, the boundaries of the clumps are not rigid. Due to the thermal bistability, material streaming onto the clump cools rapidly and thus is accreted quickly. If a clump of radius $R_{c}$ and density $n_{c}$ traveled through the opposing flow of density $n_{0}$ at a velocity $v_{c}$, then it would accrete its own mass (assuming a cross section of $\pi R_{c}^{2}$ and a constant relative velocity) after

$$
t_{\text {mix }}=\frac{3 R_{c} n_{c}}{4 v_{c} n_{0}}=79 \frac{n_{c}}{n_{0}}\left(\frac{R_{c}}{\mathrm{pc}}\right)\left(\frac{v_{c}}{\mathrm{~km} \mathrm{~s}^{-1}}\right)^{-1} \mathrm{Myr},
$$

or, for our parameters, after $t_{\mathrm{mix}} \approx 5 \mathrm{Myr}$. If the clump has accreted its own mass (of the opposing flow's metallicity), the mixing ratio (Equation (7)) will be one. The bulk of the mass is residing in the clumps, thus, once global collapse sets in, the cores are (for the most part) being assembled from the well-mixed clumps.

\section{DISCUSSION AND CONCLUSION}

We present the results of two simulations of flow-driven molecular cloud formation. The models are identical except for the physical conditions of the inflowing gas. One model ("Smooth") starts out with a completely uniform flow. The other model ("Clumpy") uses the same mass inflow rate, but the gas is distributed in dense clumps with a filling factor of $5 \%$.

In both cases, the thermal and dynamical fragmentation of the shocked collision region leads to turbulence, at a level of $10 \%-20 \%$ of the inflowing kinetic energy. The Clumpy run shows a somewhat higher velocity dispersion initially, since the clumps are less easily stopped in a flow collision (see Section 3.2).

Due to the lower compression factor in the Clumpy model, less gas is being cooled to higher densities than in the Smooth run. Thus, the kinetic energy of the inflow is less efficiently dissipated. Together with a non-contiguous distribution of cold, sub-jeans, fragments, this leads to a suppression of local collapse for nearly $20 \mathrm{Myr}$ after the initial flow collision. At that point, sufficient mass has assembled to induce global collapse of the whole region, resulting in a "star burst" (more appropriately, "sink burst") at a high sink formation rate. In contrast, the 

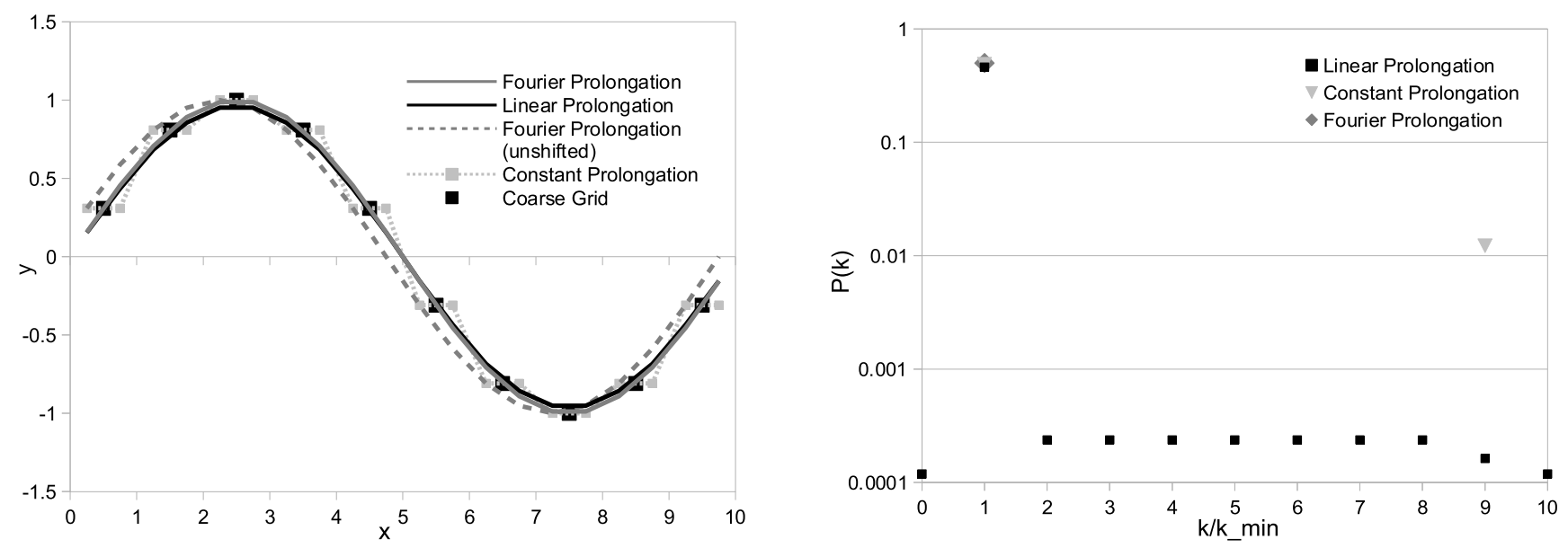

Figure 9. Left: various methods of prolongation applied to a simple sine wave. Right: corresponding power as a function of wavelength.

Smooth run shows local collapse already after $10 \mathrm{Myr}$, at less than half the sink formation rate of the Clumpy run. Thus, while the clumps lead to a higher core formation rate, they also delay the onset of core formation. We conclude that-at least within the limits of our numerical experiment-substructure in the flow increases the accumulation time necessary for gravitational collapse.

While perfectly smooth flows colliding at a geometrically slightly perturbed interface already generates turbulence in the cold slab (Smooth run; Heitsch et al. 2008b), introducing structure increases the turbulent kinetic energy. This is consistent with the findings of Dobbs \& Bonnell (2007), who modeled shocks traveling into a structured medium, and with the results of Inoue \& Inutsuka (2012), who studied the flow-driven formation of clouds with fully developed turbulent inflows.

Due to the local nature of the thermal fragmentation, more fragmentation will occur with increasing resolution (Hennebelle $\&$ Audit 2007), thus, the times quoted here are upper limits for the onset of local collapse. Nevertheless, structured flows can delay the onset of (substantial) local collapse. Global collapse is clearly visible in the Clumpy run, but seems suppressed in the Smooth run.

The differences between Clumpy and Smooth inflows extend to the mixing efficiencies. Somewhat counter to a naive expectation, the Smooth initial conditions result in less well mixed material (and cores). This is primarily due to the NTSI funneling material preferentially into the troughs located far into the opposing inflow. For the Clumpy run, the global collapse of the accumulated clumps erases all memory of the initial inflow direction.

Obviously, we have chosen two extremes as our initial conditions. It is more likely that the inflows themselves will contain turbulent velocity and density structures that are coherent in space (e.g., Hennebelle et al. 2008; Inoue \& Inutsuka 2012). Spatial coherence leads to stronger shocks in the collision region, and thus to more efficient energy dissipation. In that sense, our Clumpy run is overestimating the effect of structured inflows.

We thank the anonymous referee for an extremely thoughtful and thorough report, providing substantial food for thought. Support for this work was in part provided by NASA through awards issued by JPL/Caltech through Spitzer program 20269, the Department of Energy through grant number DE-SC0001063, Cornell University through agreement number 41843-
7012, the National Science Foundation through grants AST0807363 as well as the Space Telescope Science Institute through grants HST-AR-11250 and HST-AR-11251. We also thank the University of Rochester Laboratory for Laser Energetics and funds received through the DOE Cooperative Agreement No. DE-FC03-02NA00057. F.H. acknowledges support from NSF grant AST-0807305, NHSC 1008, and the NC Space Grant young investigator program. This research was also supported in part by the Center for Research Computing at the University of Rochester as well as the National Science Foundation through TeraGrid resources provided by the National Center for Supercomputing Applications.

\section{APPENDIX PROLONGATION}

To illustrate the technique of prolongating in Fourier space, we first consider a one-dimensional (1D) grid with 10 points over a domain of length 10 containing a sine wave labeled as "Coarse Grid" in the left panel of Figure 9. We also show the power in the corresponding spectra labeled "Fourier prolongation" in the right panel which only has a single non-zero point corresponding to the minimum wave number $k_{\min }=2 \pi / L$. We then map this coarse grid onto a finer grid comprised of 20 points and consider various methods of prolongating the coarse data.

If we use constant prolongation (also shown in the left panel of Figure 9, we introduce significant stair stepping, which produces a fair amount of power near the wavelength corresponding to the coarse grid $\left(10 k_{\min }\right)$ The additional power actually occurs at $|k|=9 k_{\min }$ (seen in the right panel of Figure 9). This is because the stair stepping is modulated by the derivative of the original function, so the constant prolongation includes a signal that is a product of two sine waves: 1 at $k_{\min }$ and another at $10 k_{\min }$ which is equivalent to the sum of two sine waves at $9 k_{\min }$ and $11 k_{\min } .11 k_{\min }$ is equivalent to $-9 k_{\min }$ for the fine mesh, since the discrete $\mathrm{fft}$ transform is the same for a wavevector of $i$ and $N-i$ and $N=20$ for the fine mesh: $\hat{f}_{i}=\sum e^{2 \pi / L I i j \Delta x} x_{j}=\sum e^{2 \pi I i j / N} x_{j}=\sum e^{2 \pi I(i-N) j / N} x_{j}$.

The linear prolongation reduces the stair stepping, but it still spreads the power over a range of higher wave numbers. The Fourier prolongation essentially performs a Fourier transform on the coarse data set, pads the higher frequencies with zeros, and then performs the inverse Fourier transform back to a finer mesh. By its construction it conservers the power spectra. However, because the coarse and fine mesh are not collocated, care must 
be taken to add the correct phase to each Fourier component before performing the inverse transform.

In 1D this works as follows: starting with an initial 1D set of $N$ evenly spaced grid points $X$, and values $Y$, we perform an FFT to get the coarse Fourier transform $F$. We then map $F$ to a finer transform $f$ by copying the values from $F$ to the position in $f$ that correspond to the same physical wavenumber:

$$
\begin{aligned}
f^{*}(1: N / 2) & =F(1: N / 2+1) \\
f^{*}(N / 2: 2 N-N / 2) & =0 \\
f^{*}(2 N-N / 2+2: 2 N) & =F(N / 2+2: N) .
\end{aligned}
$$

If we were to transform $f^{*}$ back to physical space, we would get the right function, though it would be offset due to the coarse and fine cells not being collocated (see the left panel of Figure 9.) To correct this we add to each component a phase corresponding to the wavenumber $k$ times the offset between the coarse and fine cells $\Delta x_{c} / 4=\Delta x_{f} / 2$ :

$$
f(i)=f^{*}(i) e^{I i k_{\min } \frac{\Delta x_{c}}{4}}=f^{*}(i) e^{\frac{2 \pi I}{4 N}} .
$$

We then transform back to the finer mesh using an inverse FFT (IFFT), i.e., $y=\operatorname{IFFT}(f)$. We can then update the finer mesh with data from that level, and then repeat the procedure down to the finest level. This will produce a spectra with minimum noise due to the various grid sizes used in constructing the power spectra. It is somewhat straightforward to extend this idea to multiple dimensions.

\section{REFERENCES}

Audit, E., \& Hennebelle, P. 2005, A\&A, 433, 1

Ballesteros-Paredes, J., \& Hartmann, L. 2007, RMxAA, 43, 123

Ballesteros-Paredes, J., Hartmann, L., \& Vázquez-Semadeni, E. 1999, ApJ, 527,285

Ballesteros-Paredes, J., Hartmann, L. W., Vázquez-Semadeni, E., Heitsch, F., \& Zamora-Avilés, M. A. 2011, MNRAS, 411, 65

Banerjee, R., Vázquez-Semadeni, E., Hennebelle, P., \& Klessen, R. S. 2009, MNRAS, 398, 1082
Burkert, A., \& Hartmann, L. 2004, ApJ, 616, 288

Carroll-Nellenback, J. J., Shroyer, B., Frank, A., \& Ding, C. 2013, JCoPh, 236, 461

Dobbs, C. L., \& Bonnell, I. A. 2007, MNRAS, 374, 1115

Evans, N. J., II, Dunham, M. M., Jørgensen, J. K., et al. 2009, ApJS, 181,321

Falgout, R., \& Yang, U. 2002, in Computational Science - ICCS 2002, ed. P. Sloot et al. (Berlin: Springer), 632

Federrath, C., Banerjee, R., Clark, P. C., \& Klessen, R. S. 2010, ApJ, 713,269

Gardiner, T. A., \& Stone, J. M. 2008, JCoPh, 227, 4123

Hartmann, L., Ballesteros-Paredes, J., \& Bergin, E. A. 2001, ApJ, 562, 852

Hartmann, L., \& Burkert, A. 2007, ApJ, 654, 988

Heitsch, F., Ballesteros-Paredes, J., \& Hartmann, L. W. 2009, ApJ, 704, 1735

Heitsch, F., Burkert, A., Hartmann, L. W., Slyz, A. D., \& Devriendt, J. E. G. 2005, ApJL, 633, 113

Heitsch, F., Hartmann, L. W., \& Burkert, A. 2008a, ApJ, 683, 786

Heitsch, F., Hartmann, L. W., Slyz, A. D., Devriendt, J. E. G., \& Burkert, A. 2008b, ApJ, 674, 316

Heitsch, F., Slyz, A. D., Devriendt, J. E. G., Hartmann, L. W., \& Burkert, A. 2006, ApJ, 648, 1052

Hennebelle, P., \& Audit, E. 2007, A\&A, 465, 431

Hennebelle, P., Banerjee, R., Vázquez-Semadeni, E., Klessen, R. S., \& Audit, E. 2008, A\&A, 486, L43

Hueckstaedt, R. M. 2003, NewA, 8, 295

Inoue, T., \& Inutsuka, S.-i. 2008, ApJ, 687, 303

Inoue, T., \& Inutsuka, S.-i. 2009, ApJ, 704, 161

Inoue, T., \& Inutsuka, S.-i. 2012, ApJ, 759, 35

Klein, R. I., McKee, C. F., \& Colella, P. 1994, ApJ, 420, 213

Koyama, H., \& Inutsuka, S.-i. 2002, ApJL, 564, L97

Larson, R. B. 1985, MNRAS, 214, 379

McKee, C. F., \& Ostriker, E. C. 2007, ARA\&A, 45, 565

Pon, A., Johnstone, D., \& Heitsch, F. 2011, ApJ, 740, 88

Pringle, J. E., Allen, R. J., \& Lubow, S. H. 2001, MNRAS, 327, 663

Vázquez-Semadeni, E., Colín, P., Gómez, G. C., Ballesteros-Paredes, J., \& Watson, A. W. 2010, ApJ, 715, 1302

Vázquez-Semadeni, E., Gómez, G. C., Jappsen, A.-K., Ballesteros-Paredes, J., \& Klessen, R. S. 2009, ApJ, 707, 1023

Vázquez-Semadeni, E., Gómez, G. C., Jappsen, A. K., et al. 2007, ApJ, 657,870

Vázquez-Semadeni, E., Passot, T., \& Pouquet, A. 1995, ApJ, 441, 702

Vázquez-Semadeni, E., Ryu, D., Passot, T., González, R. F., \& Gazol, A 2006, ApJ, 643, 245

Vishniac, E. T. 1994, ApJ, 428, 186 